reports between 1968-1989 in a review paper from the Division of Child Neurology, The Children's Hospital, Philadelphia and the Department of Neurology, Children's National Medical Center, George Washington University, Washington, DC. The increasing use of neuropsychological testing and the development of prospective studies during the first half of the $1980 \mathrm{~s}$ has shown that from $40 \%$ to $100 \%$ of long-term survivors have some form of cognitive deficit in various intelligence quotients, visual/perceptual skills, learning abilities, and adaptive behavior. More cognitive deficits are detected the larger the time lapse from treatment to the testing evaluation. Factors predictive of subsequent cognitive deficits included a younger age at diagnosis, radiotherapy, methotrexate chemotherapy, and tumor location. Cognitive deficits occurred more frequently when medulloblastomas adhered to the brain stem, when tumors extended to the hypothalamus, and with hemispheric tumors. Surgery and preoperative hydrocephalus did not appear to modify cognition. (Glauser TA, Packer RJ. Cognitive deficits in long-term survivors of childhood brain tumors. Child's Nerv Syst Feb $1991 ; 7: 2-12)$.

COMENT. The quality of life for long-term survivors of childhood brain tumors becomes more important as improvements in treatment are developed. Long-term follow-up with neuropsychological tests is necessary since significant progressive deterioration in cognitive function may be demonstrated as the testing interval following treatment increases. (Duffner PK et al. Ann Neurol 1988; 23:575).

\title{
STROKE AS LATE SEQUELLA OF CRANIAL IRRADIATION
}

The development of cerebrovascular disease manifested by strokes or transient ischemic attacks six months to four years after treatment of brain tumors in 11 children is reported from the Children's Hospital of Los Angeles, University of Southern California School of Medicine, Los Angeles, CA. All patients had received radiation therapy and seven chemotherapy. One patient, aged 4, with an incompletely resected craniopharyngioma, developed a right hemiparesis two years after treatment followed by left-sided focal motor seizures with quick progression to decerebrate posturing, central hyperventilation, coma, and death. Autopsy revealed bilateral cerebral infarctions as well as diffuse gliosis. Branches of the carotid artery showed severe thickening with subintimal proliferation of connective tissue. Smaller vessels showed cellular intimal proliferation with areas of leukocytic infiltration. (Mitchell WG et al. Stroke as a late sequela of cranial irradiation for childhood brain tumors. J Child Neurol April 1991; $6: 128-133$ ).

COMMENT. Damage to medium and large intracranial vessels in addition to small vessel damage may result in late onset of stroke or transient ischemic attacks caused by radiation and chemotherapy in children with brain tumors. The cambination of methotrexate and cranial irradiation is more likely to produce a leukoencephalopathy than either treatment alone. The addition of chemotherapy may potentiate the damage to endothelial cells produced by radiation (mineralizing microangiopathy). 